$\mathrm{MAN} / \mathrm{HEP} / 2009 / 20$

MCnet/09/20

DESY 09-214

Dec 2009

\title{
Real Emission and Virtual Exchange of Gravitons and Unparticles in Pythia8
}

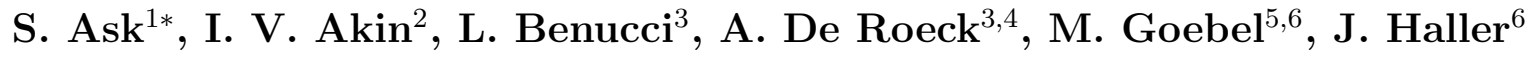 \\ 1) University of Manchester, UK. \\ 2) Middle East Technical University, Ankara, Turkey. \\ 3) Universiteit Antwerpen, Belgium. \\ 4) CERN, Geneva, Switzerland. \\ 5) DESY, Hamburg, Germany. \\ 6) Universität Hamburg, Germany. \\ * E-mail: Stefan.Ask@manchester.ac.uk
}

\begin{abstract}
Models with large extra dimensions as well as unparticle models could give rise to new phenomena at collider experiments due to real emission or virtual exchange of gravitons or unparticles. In this paper we present the common implementation of these processes in the Monte Carlo generator Pythia8, using relations between the parameters of the two models. The program offers several options related to the treatment of the UV region of the effective theories, including the possibility of using a form factor for the running gravitational coupling. Characteristic results obtained with PyтHIA8 have been used to validate the implementations as well as to illustrate the key features and effects of the model parameters. The results presented in this paper are focused on mono-jet, di-photon and di-lepton final states at the LHC.
\end{abstract}




\section{Introduction}

Models with large extra dimensions (LED) are popular extensions of the Standard Model (SM) and studies of LED phenomena are usually based on the so-called ADD scenario [1], where gravity alone has access to the extra dimensions. The large size of the extra dimensions gives rise to dense Kaluza-Klein (KK) mass modes which appear as a continuous graviton mass spectrum. Due to the large number of contributing mass states graviton processes could obtain sufficiently large cross sections to be observed at collider experiments, where the new processes involve real emission or virtual exchange of gravitons. These processes have been studied in great detail [2], however, no signs of new physics have been indicated at existing experiments.

More recently so-called unparticle models [3] have also gained a large interest. These models relate to physics originating from a scale invariant new sector which is coupled to the SM through a connector sector with a high mass scale. Unparticle models can often, at least from a phenomenological point of view, be associated with extra dimension models and the particular case addressed here would result in phenomena very similar to the scenario of LED gravity. Having access to processes with the same final state from more than one model can be helpful to generalise the experimental analysis as well as for studying differences in the measured observables. The latter, in turn, could also be of value after a discovery to characterise the observed signal.

This paper describes the processes involving real emission or virtual exchange of unparticles $(\mathcal{U})$ or LED gravitons $(G)$ as implemented in PythiA8 [4]. The corresponding processes are:

- Mono-jets $(\mathcal{U} / G+j e t): g g \rightarrow \mathcal{U} / G+g, q g \rightarrow \mathcal{U} / G+q, q \bar{q} \rightarrow \mathcal{U} / G+g$;

- Mono- $Z / \gamma(\mathcal{U} / G+Z / \gamma): f \bar{f} \rightarrow \mathcal{U} / G+Z, f \bar{f} \rightarrow \mathcal{U} / G+\gamma$;

- Di-photons $(\gamma \gamma): f \bar{f} \rightarrow \mathcal{U}^{*} / G^{*} \rightarrow \gamma \gamma, g g \rightarrow \mathcal{U}^{*} / G^{*} \rightarrow \gamma \gamma$;

- Di-leptons $(\ell \bar{\ell}): f \bar{f} \rightarrow \mathcal{U}^{*} / G^{*} \rightarrow \ell \bar{\ell}, g g \rightarrow \mathcal{U}^{*} / G^{*} \rightarrow \ell \bar{\ell}$.

Because of the large similarities both models can be covered by a common implementation [5], where only a simple translation of a few model related constants is required to change between the models. In addition to technical advantages, this common description makes comparisons between the processes both consistent and transparent. The effective theory description of these processes behaves poorly in the UV limit and several related options are available in the program. These include a form factor [6] for the graviton processes which represents a realistic alternative of the behaviour at high energies due to the running of the gravitational coupling.

The Pythia8 implementations have been validated against the original theory papers and we present here some of these results. Besides providing reference results for the PYTHIA 8 program, the results illustrate different characteristic effects of the model parameters and various key features ${ }^{1}$. All results are produced with respect to the nominal LHC conditions ( $p p$-collisions at a centre-of-mass energy of $\sqrt{s}=14 \mathrm{TeV}$ ), unless explicitly stated otherwise.

This paper is organised as follows. The first section starts with a description of the model parameters and different conventions. It is followed by a description of the implementation of the processes and the relations between the two models. This section also describes the available options for treating the UV region of the effective theories. Sec. 3 to 5 present the

\footnotetext{
${ }^{1}$ In order to prevent clutter, the figures presented in this paper do not include statistical errors, which should not be significant for the conclusions.
} 
different program options in PYтніA8 together with results related to the individual processes: $\mathcal{U} / G+j e t, \gamma \gamma$, and $\ell \bar{\ell}$ production. In addition, Sec. 5 draws special attention to possibile effects on the forward-backward asymmetry in lepton pair production from spin-1 unparticle exchange. The $\mathcal{U} / G+Z / \gamma$ processes are not addressed here since they were already presented in a previous paper [5]. A complete list of the process and model parameter names used in the PYTHIA8 program can be found in the appendix. The appendix also contains complementary information used for the scalar unparticle process, $f \bar{f} \rightarrow Z \mathcal{U}$, which is not available in the literature.

This paper presents results obtained with PYTHIA version 8.125 and all parameters not explicitly mentioned in the text have been kept at the default values of this version. During the work a few minor corrections have been made $^{2}$, which all are included in the following version 8.130. In order to produce results comparable to what is available in the literature, only the hard part of the process has been considered ${ }^{3}$ and the MRST2001 parton distribution functions (PDF) of the proton have been used unless stated otherwise.

\section{Implementation in Pythia8}

The common implementation of the LED graviton and unparticle processes in PYTHIA8 is generally based on the cross section expressions derived for the unparticle case. These expressions can be converted into the corresponding LED graviton formulae by simple translations of a few model related constants. For this reason we first discuss the unparticle process and afterwards we introduce the translation into its LED equivalent. The values of the model parameters are sometimes constrained by arguments valid in different scenarios of the model, however, in order to allow for more phenomenologically motivated studies the implementations in PYTHIA8 generally allow for any parameter value that is technically possible. Particular model constraints therefore have to be respected explicitly by the users. As mentioned above, the common implementation of the $\mathcal{U} / G+Z / \gamma$ processes was originally presented in [5], together with a study of $U / G+Z$ production at the LHC, but for completeness relevant parts related to these processes will be repeated here.

\subsection{Parameter Conventions}

The parameter convention used here for the unparticle case follows [7]. It is assumed that any potential gaps in the unparticle mass spectrum are sufficiently small not to affect the high energy behaviour at particle colliders and that the life time is long enough to prevent decays at distances relevant for collider experiments. The main parameters are a scale dimension parameter $\left(d_{\mathcal{U}}\right)$, an unparticle renormalisation scale $\left(\Lambda_{\mathcal{U}}\right)$ and a coupling constant $(\lambda)$ of the unparticles to the SM fields, which is related to the mass scale of the connector sector.

In principle there are restrictions on the allowed ranges of the parameters. Full conformal invariance restricts the scaling dimension of the unparticle to $d_{\mathcal{U}}>1$ (scalar), $d_{\mathcal{U}}>3$ (vector) and $d_{\mathcal{U}}>4$ (tensor), which follow from Mack's unitary constraint, as discussed for example in [8]. By assuming scale invariance, but not full conformal invariance, these restrictions are relaxed. In this paper we take a phenomenological approach and allow for the full $d_{\mathcal{U}}$ range and

\footnotetext{
${ }^{2}$ The corrections are related to the $\ell \bar{\ell}$ process and, in addition, the spin- 0 case of the $\mathcal{U}+Z / \gamma$ process has been added.

${ }^{3}$ Where the program parameters PartonLevel:MI, PartonLevel:ISR and PartonLevel:FSR have been turned off.
} 
the lowest bound $d_{\mathcal{U}}>1$ is used as the overall lower limit in Pythia8. For some processes it is, however, necessary to constrain this parameter to the range $1<d_{\mathcal{U}}<2$ for numerical reasons. For $d_{\mathcal{U}}>2$, especially in the case of virtual unparticle exchange, contributions that depend on the UV completion of the theory are relevant which suggests that the effective theory used will not be valid. The ranges allowed in PYTHIA8 are in accordance with [7, 9], where for example Eq. (4) in the latter paper shows that the propagator diverges ${ }^{4}$ when $d_{\mathcal{U}} \rightarrow 2$,

$$
\begin{array}{r}
\int d^{4} x e^{i P x}\left\langle 0\left|T O_{\mathcal{U}}^{\kappa}(x) O_{\mathcal{U}}^{\kappa}(0)\right| 0\right\rangle \\
\propto \frac{A_{d_{\mathcal{U}}}}{2 \sin \left(d_{\mathcal{U}} \pi\right)}=\chi_{\mathcal{U}} \\
A_{d_{\mathcal{U}}}=\frac{16 \pi^{2} \sqrt{\pi}}{(2 \pi)^{2 d_{\mathcal{U}}}} \frac{\Gamma\left(d_{\mathcal{U}}+\frac{1}{2}\right)}{\Gamma\left(d_{\mathcal{U}}-1\right) \Gamma\left(2 d_{\mathcal{U}}\right)}
\end{array}
$$

where $A_{d_{\mathcal{U}}}$ is a normalisation constant related to the rather peculiar unparticle phase space. Hence, this divergence implies that the virtual $\mathcal{U}$ exchange processes in PyтHIA 8 require $d_{U}<2$. The formulae obtained for the $\mathcal{U}$ emission processes on the other hand give finite results also for the range $d_{\mathcal{U}}>2$. However, in this case the results have to be interpreted more carefully as discussed in Sec. 2.3.

The parameter convention used here for LED gravity follows [11]. The gaps between the individual KK modes of the graviton are determined by the size of the extra dimensions together with a possible curvature of space-time, like in the so-called Randall-Sundrum scenarios [12]. It has been shown that a small curvature can remove cosmological constraints from low energy processes [13]. However, for the processes addressed here it is assumed that any mass gaps in the graviton mass spectrum are sufficiently small for the high energy phenomena to be unaffected.

The main model parameters correspond to the fundamental scale of $D(=n+4)$ dimensional gravity $\left(M_{D}\right)$, the number of extra dimensions $(n)$ and in the case of virtual graviton exchange an effective cut-off scale $\left(\Lambda_{T}\right)$. The extra dimensions are normally assumed to form a $n$-dimensional torus with a common radius $\left(R_{D}\right)$. This radius is determined by $n, M_{D}$ together with the 4 dimensional Planck mass and is for this reason not included as a parameter for the processes in Pythia8. A number of different conventions are used in the literature and the most popular are related as follows:

- GRW [11]: $M_{D}, R_{D}, n, \Lambda_{T}$ (used in Pythia8);

- $\operatorname{MPP}[14]: M^{n+2}=2 M_{D}^{n+2}$;

- $\operatorname{HLZ}[15]: M_{S}^{n+2}=8 \pi^{1-\frac{n}{2}} \Gamma\left(\frac{n}{2}\right) M_{D}^{n+2}, R_{S}=2 \pi R_{D}$;

- $\operatorname{HR}[6]: \Lambda_{H}^{4}=\frac{2}{\pi} \Lambda_{T}^{4}$.

\subsection{Implementation of the Processes}

The processes in PythiA8 are available for unparticles with spin 0, 1 and 2. However, not all spin values are available for all processes. The $\mathcal{U} / G+Z / \gamma$ processes are implemented as $2-$ to -2 parton level processes based on the $Z$ matrix elements (ME) calculated in [7] for spin- 1 and spin-2 unparticle emission. In addition the spin-0 case is available, which is based on the ME given in the appendix. In the case of $Z$ production, no $Z / \gamma^{*}$ interference is included and the $Z$ decays isotropically. The photon process corresponds to the photon limit of the $Z$ process.

\footnotetext{
${ }^{4}$ The relation between $d_{\mathcal{U}}$ and the UV sensitivity has for example been studied in [10].
} 
All $\mathcal{U} / G$ emission processes implemented in PyTHIA8 share the graviton particle code 5000039, since the $\mathcal{U}$ or $G$ has a continuous mass spectrum. This is in contrast to processes related to an individual mass state, where for example the first KK mode of the graviton would have the particle code 5100039. The main difference with respect to existing processes in PYTHIA8 was the continuous mass spectrum of the unparticle. This has been solved by generating unparticle masses, using already existing Pythia8 functionality, according to a Breit-Wigner distribution. The events are then re-weighted in order to obtain a correct mass distribution, as given by the differential cross section. For this reason the Breit-Wigner shape should be matched to the relevant range of the mass spectrum in order to achieve the highest possible Monte Carlo (MC) efficiency and generation speed. The Breit-Wigner shape is defined by the program parameters, 5000039:m0, 5000039:mWidth, 5000039:mMin, 5000039:mMax, according to the standard particle data scheme in PүтніА8.

The $\mathcal{U} / G+$ jet processes have been implemented in the same way as the $\mathcal{U} / G+Z / \gamma$ processes. They are available for spin-0 and spin-1 unparticles, however, graviton emission is the only available spin-2 scenario. The processes are based on MEs from [11] for $G$ emission and [7, 16] for the $\mathcal{U}$ processes. The spin- 0 unparticle interactions used for the processes presented here are given by the effective operators [7],

$$
\mathcal{L} \supset \frac{\lambda}{\Lambda_{\mathcal{U}}^{d_{\mathcal{U}}-1}} \sum_{f} \bar{f} f O_{\mathcal{U}}+\frac{\lambda}{\Lambda_{\mathcal{U}}^{d_{\mathcal{U}}}} \sum_{G} G_{\alpha \beta} G^{\alpha \beta} O_{\mathcal{U}}
$$

where $f$ refers to fermions and $G_{\alpha \beta}$ to gauge bosons. Both terms in Eq. (3) lead to amplitudes at leading order for, $q \bar{q} \rightarrow \mathcal{U} / G+g$ and $q g \rightarrow \mathcal{U} / G+q$, whereas $g g \rightarrow \mathcal{U} / G+g$ only involves the gluon vertex, i.e. the second term. Since the gluon vertex is suppressed by one additional power of $\Lambda_{\mathcal{U}}$ compared to the fermion vertices, i.e. two powers with respect to the cross section, the MEs of the two quark initiated processes are approximated by including only diagrams with unparticle emission from a fermion line. The coupling constants of the two terms are not necessarily the same and it could be of interest to study them separately. Again due to the suppression by different powers of $\Lambda_{\mathcal{U}}$ in the two terms, this can be approximated by using $q \bar{q} \rightarrow \mathcal{U} / G+g$ and $q g \rightarrow \mathcal{U} / G+q$ to represent the contributions from the quark vertices and $g g \rightarrow \mathcal{U} / G+g$ to represent the gluon vertex contribution.

In order to obtain the corresponding graviton emission processes, the unparticle parameters have been interpreted as follows,

$$
\begin{array}{r}
r=\lambda_{2}^{\prime} / \lambda_{2}=1 \\
d_{\mathcal{U}}=\frac{n}{2}+1 \\
\Lambda_{\mathcal{U}}=M_{D} \\
A_{d_{\mathcal{U}}} \rightarrow S^{\prime}(n)=\frac{2 \pi \pi^{n / 2}}{\Gamma\left(\frac{n}{2}\right)}
\end{array}
$$

where $\lambda_{2}$ and $\lambda_{2}^{\prime}$ refer to the possibility of having different couplings related to the two effective spin-2 unparticle operators [7]. This translation is automatically done inside PYTHIA 8 when the graviton processes are used.

The virtual $\mathcal{U} / G$ exchange processes are implemented in PүтніA 8 as 2-to-2 parton level processes. Certain processes contain coherent SM amplitudes and converge to the SM results if the unparticle contribution is close to zero, e.g. by setting $\lambda=0$ or $\Lambda_{\mathcal{U}} \rightarrow \infty$. The SM results 
shown for comparison in the figures below have been obtained by the corresponding $\mathcal{U} / G$ process using such extreme parameter values.

The $\gamma \gamma$ processes are available for spin- 0 and spin- 2 unparticles based on the matrix elements in $[7,9]$. In the spin-2 case, the fermion initiated process includes the corresponding SM $t$-channel process (equivalent to PromptPhoton:ffbar2gammagamma) together with the related interference. The spin-2 gluon process does not involve the possible SM box diagram which for this reason must be included separately, e.g. using the PYTHIA 8 process ${ }^{5}$ PromptPhoton: gg2gammagamma.

The $\ell \bar{\ell}$ processes only concern charged lepton pair production. The fermion initiated process is available for both spin- 1 and spin-2 unparticles and includes the $\mathrm{SM} Z / \gamma^{*}$ amplitudes and interference. The gluon process is only available for spin-2 and does not contain any SM contributions. All processes are implemented following the MEs in [7]. Since the fermion initiated process could be used for studies at lepton colliders, it should be noted that currently no SM $t$-channel diagram is included in PYTHIA8. For example, this would be relevant for studies of Bhabha scattering. The SM contribution should therefore be similar to the PyTHIA 8 process, WeakSingleBoson : ffbar2gmZ, when only the charged lepton final states are considered. A spin1 unparticle can have chiral couplings to fermions which could lead to interesting interference effects [17]. For this reason the chiral properties of the coupling can be specified explicitly by two coupling parameters $\left(g_{X X}, g_{X Y}\right)$. These can be assigned the values 1,0 or -1 which are multiplied with the different helicity amplitudes, $X X=L L / R R$ and $X Y=L R / R L$. This implies that a vector coupling corresponds to, $g_{X X}=g_{X Y}=1$, and an axial coupling to, $g_{X X}=1, g_{X Y}=-1$.

The corresponding graviton processes, where the unparticle is replaced by a virtual graviton, are again obtained using the common implementation. This has been achieved by the same approach as for the $\mathcal{U} / G$ emission processes where two constants had to be adjusted. The cross sections given by the spin-2 unparticle MEs discussed above become identical to the graviton cross section expressions in [11] when the unparticle parameters are translated as follows,

$$
\begin{gathered}
d_{\mathcal{U}}=2 \\
\chi_{\mathcal{U}}=1 \\
\lambda=\sqrt{4 \pi} \\
\Lambda_{\mathcal{U}}=\Lambda_{T}
\end{gathered}
$$

The virtual graviton exchange processes are UV sensitive, similar to the unparticle scenario. The graviton amplitudes are dominated by contributions that depend on the UV completion of the theory when the number of large extra dimensions ${ }^{6}, n>2$. Since this scenario is generally of great interest, the UV sensitive part is commonly replaced by an arbitrary cut-off scale, which parametrises our ignorance and removes the $n$ dependence.

\subsection{Treatment of the Effective Theory at High Energies}

Since the current limits on the effective mass scales related to the different processes $\left(\Lambda_{\mathcal{U}}, M_{D}\right.$ and $\left.\Lambda_{T}\right)$ are well below the possible center-of-mass energies of the hard process, $\hat{s}$, at the LHC, a number of different options are available for the case where the hard scale of the process

\footnotetext{
${ }^{5}$ Note that this process is based on a massless approximation which can be relevant.

${ }^{6}$ Which have a similar role to $d_{\mathcal{U}}$ in the unparticle model.
} 
approaches or exceeds the scale of validity of the effective theory. These are specified by the program parameter CutOffmode, which can take the following values:

- CutOffmode = 0: Include all $\hat{s}$ values;

- CutOffmode = 1: Suppress the cross section for $\hat{s}>\Lambda_{\mathcal{U}}^{2}\left(M_{D}^{2}\right)$;

- CutOffmode = 2: Gravity form factor, using SigmaProcess: renormScale2;

- CutOffmode = 3: Gravity form factor, using $\mu=E_{j e t / Z / \gamma}^{*}$.

The default option (CutOffMode $=0$ ) implies no restrictions, but includes the cross section contribution for any $\hat{s}$ value. The first alternative option (CutOffMode $=1$ ) simply suppresses the cross section [18] by a factor $\Lambda_{\mathcal{U}}^{4} / \hat{s}^{2}$ at $\hat{s}$ values that exceed the mass scale of the effective theory, $\Lambda_{\mathcal{U}}^{2}$ or $M_{D}^{2}$. This truncation of the cross section also implies that the $\mathcal{U} / G$ mass spectrum is suppressed at larger values. The $\mathcal{U} / G$ mass spectrum becomes increasingly peaked towards larger values with increasing $n$ or $d_{\mathcal{U}}$. For this reason, the suppression effect generally becomes more significant for increased values of these parameters. In a similar way the effect of this truncation becomes larger with an increased transverse energy requirement in the analysis. This option is only implemented for the $\mathcal{U} / G$ emission processes and further details can be found in [5]. Starting the truncation directly above the effective mass scale can often be considered as conservative, where for example [11] estimates the effective theory for the LED graviton processes to be perturbative up to about $7 M_{D}$. This rather crude method of truncating the cross section was primarily intended to be used for verifying that the truncated part, which corresponds to the region where the effective theory might not be valid, has a negligible effect on the total cross section. However, in the case where it is not negligible the truncated value could also be used as a conservative estimate.

The possibility of using a form factor [6] for the gravitational coupling has also been implemented,

$$
F(\mu ; t, M, n)=\left[1+\left(\frac{\mu^{2}}{t^{2} M^{2}}\right)^{1+n / 2}\right]^{-1}
$$

Here $\mu$ is a renormalisation scale and $t$ is a $\mathcal{O}(1)$ free parameter which relates to the unknown details of the running of the gravitational coupling. The parameter $M$ is associated with the cut-off scale of the effective theory $\left(M_{D}\right.$ or $\Lambda_{T}$ depending on the process). The form factor leads to a weaker gravitational coupling at higher energies and this provides a realistic alternative of the behaviour when $\hat{s}$ approaches $M^{2}$. For both real emission and virtual exchange of gravitons the renormalisation scale $\mu$ can be specified (CutOffMode $=2$ ) to follow the choice made by the program parameter, SigmaProcess:renormScale2. For graviton emission it is possible to set the renormalisation scale equal to the $j e t / Z / \gamma$ centre-of-mass energy (CutOffMode $=3$ ) which was used in [6]. It is argued in [6] that the form factor can generally prevent virtual graviton exchange amplitudes from violating unitarity by requiring $t<2$, which is suggested as an upper bound on this parameter.

\section{Real Emission, Mono-Jets}

Both the LED graviton and unparticle models can lead to final states with a single jet plus missing transverse energy, with balancing transverse momenta. The related $G$ emission processes 


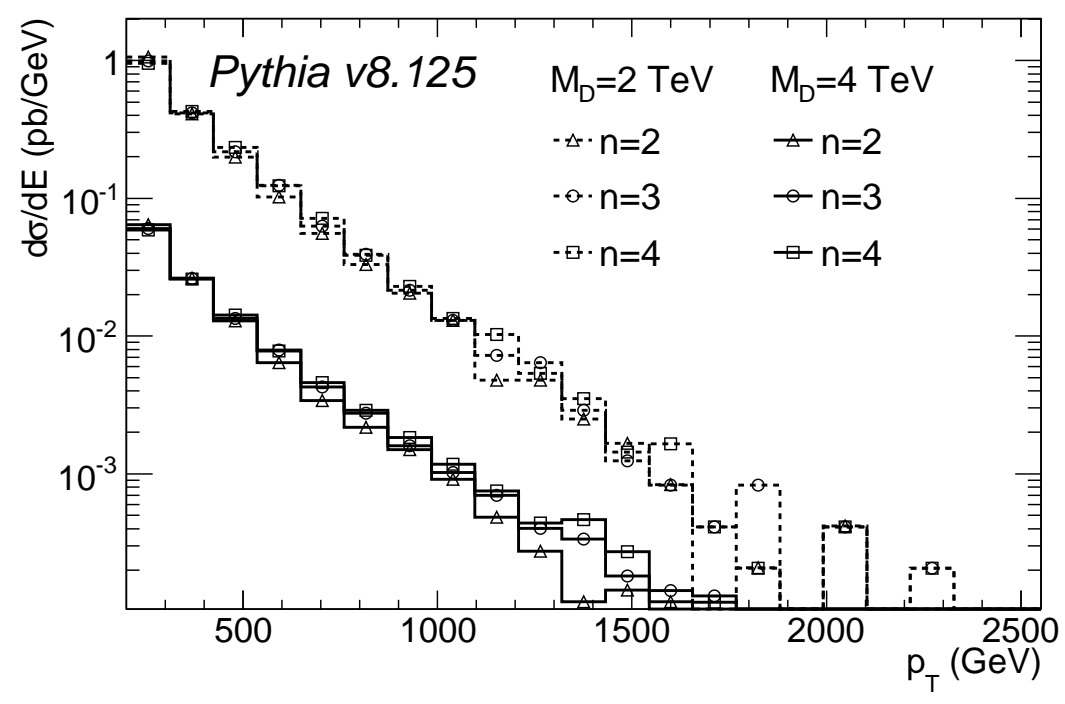

Figure 1: Differential cross section of $G+$ jet production at the LHC $(\sqrt{s}=14 \mathrm{TeV})$ as a function of transverse jet momentum. All shapes are normalised to the case $n=2$.

available in PYтнiA 8 consist of qqbar2Gg, qg2Gq, gg2Gg, with the program parameters $\mathrm{n}$, MD, CutOffmode and $t$. The corresponding $\mathcal{U}$ emission processes are qqbar2Ug, qg2Uq, gg2Ug, with the relevant parameters spinU, dU, LambdaU, lambda and CutOffmode. The unparticle processes are only available for the spin values spinU $=0,1$ and, in both the case of $G$ and $\mathcal{U}$ emission, the user should match the generated mass distribution to the cross section, as discussed in section 2.2 .

This section presents characteristic results related to $\mathcal{U} / G+$ jet production at the LHC obtained using Pythin8. The processes are validated with respect to the results presented in $[7,11,16]$ and, to allow for easy comparison, most of the selection cuts and model parameter values have been used consistently with these papers.

\subsection{LED Graviton Emission}

In Fig. 1 the differential cross section of $G+$ jet production at the LHC is shown as a function of the transverse jet momentum for different values of $M_{D}$ and $n$. For these generator level results the jet simply refers to the outgoing quark or gluon in the hard interaction. The jet has been required to have transverse momentum $p_{T}>200 \mathrm{GeV}$ together with a pseudo-rapidity $|\eta|<2$. For the large $\hat{s}$ region the form factor (c.f. Eq. (12)) has been used (CutOffMode $=2$, renormScale2 $=1$ ) with $t=1$. In order to simplify shape comparisons all distributions have been normalised to the cross section for $n=2$. As expected, changing one of the two parameters has a small effect on the shape of the different distributions. The results are consistent, both in terms of shape and cross section, with previous studies. The same distributions have also been produced using different PDFs: MRST2001, CTEQ6L, GRV94L ${ }^{7}$. As expected, the effect of using different PDFs is very small.

\footnotetext{
${ }^{7}$ Although GRV94L is relatively old it has been included since it was used in some of the early graviton papers.
} 


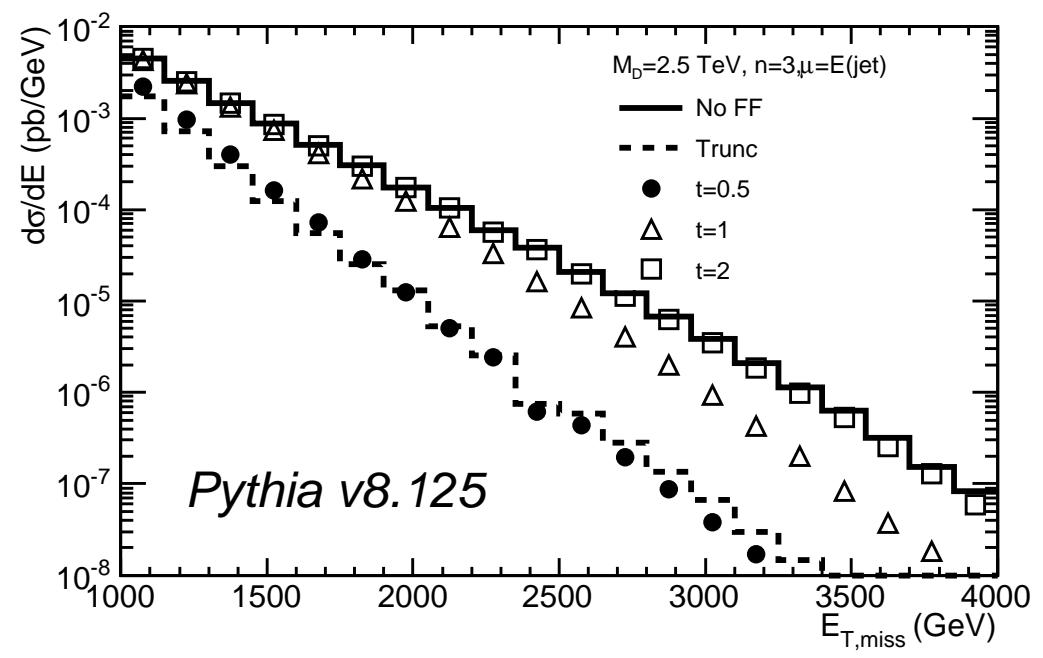

Figure 2: Differential $G+$ jet cross section at the LHC $(\sqrt{s}=14 \mathrm{TeV})$ as a function of the missing transverse energy. The plot shows results obtained using the form-factor compared with the case where all $\hat{s}$ values contribute (NoFF) and the case where the cross section is truncated (Trunc).

Since the high energy collider signals can be sensitive to the large $\hat{s}$ region, where the effective theory is not necessarily valid, it is interesting to study the effect of the different methods to treat this region (c.f. Sec. 2.3). In order to use the form factor, the quantity $\mu$ needs to be related to a physical scale in the production process and the choice of scale can affect the cross sections significantly. For the mono-jet studies presented here, the $\mu$ parameter has either been set to the jet energy (CutOffMode $=3$ ) or alternatively to the jet $p_{T}$ (CutOffMode $=2$, renormScale 2 $=1$ ).

Fig. 2 shows the missing transverse energy distributions from $G+$ jet production when using the different options related to the UV region. The form factor results are compared with the case where all $\hat{s}$ values contribute (NoFF) and the case where the cross section is truncated (Trunc). The effect of the form factor, which implies a softer distribution, is visible together with the expected dependence on the model parameters. The effect decreases with increasing $t$, where $t=2$ is shown to be similar to the NoFF distribution and the case with $t=0.5$ approaches the truncated distribution. In addition, the effect increases slightly for $\mu=E$ (jet) compared to $\mu=p_{T}$ (jet). The study showed moderate sensitivity to $n$ and the effect becomes larger for smaller values of $M_{D}$, which is consistent with Eq. (12).

\subsection{Unparticle Emission}

Fig. 3 shows the differential cross section for mono-jet production due to $\mathcal{U}$ emission at the LHC as a function of the jet energy. In accordance with [7] the cross sections correspond to spin-1 unparticle emission through the two processes, $q \bar{q} \rightarrow \mathcal{U} / G+g$ and $q g \rightarrow \mathcal{U} / G+q$ together with spin-0 unparticle emission due to the gluon process, $g g \rightarrow \mathcal{U} / G+g$. The distributions are shown for a number of $d_{\mathcal{U}}$ values. The parameters $\Lambda_{\mathcal{U}}=1 \mathrm{TeV}$ and $\lambda=1$ have been used for both the scalar and vector processes. Only jets with transverse momenta larger than $100 \mathrm{GeV}$ and with rapidities $|y(j e t)|<2$ have been considered. In addition to the cross sections predicted for 


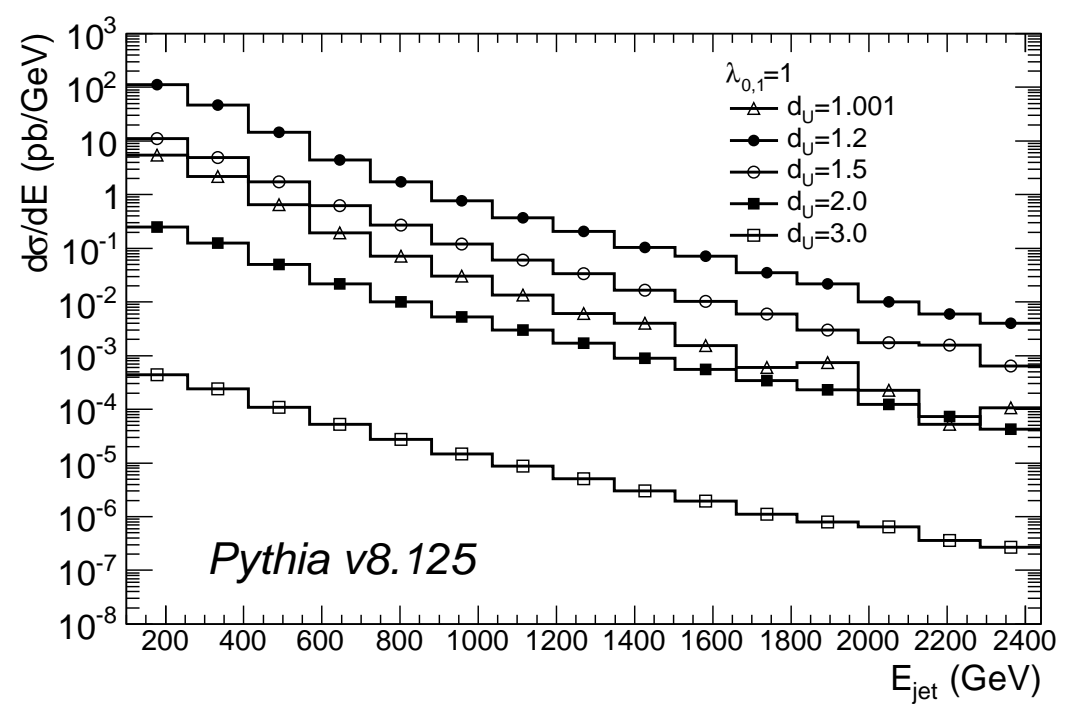

Figure 3: Differential cross section of $U+$ jet production at the LHC $(\sqrt{s}=14 \mathrm{TeV})$ as a function of the jet energy. Contributions from spin- 0 and spin- 1 unparticles are included.

these unparticle scenarios the plot demonstrates the generally small influence of the non-integer dimension parameter $d_{\mathcal{U}}$ on the slope. Both the distribution shape and cross section values have been found to be consistent with the results in the reference literature.

The coherent implementation of the similar $\mathcal{U}$ and $G$ processes in PyтнiA8 simplifies studies of the possibility to distinguish between the two models. As an example, a comparison of the differential cross section as a function of the transverse jet energy is shown in Fig. 4. The graviton distributions for two values of $n$ are compared to the distributions from spin- 1 unparticle emission for $d_{\mathcal{U}}=1.1,1.5$ and 1.9. The unparticle results contain both the two allowed sub-processes and the parameters values $\Lambda_{\mathcal{U}}=1 \mathrm{TeV}$ and $\lambda=1$ have been used. It is clearly visible that the two models exhibit different spectrum slopes almost independently of $n$ or $d_{\mathcal{U}}$ and similar results were found for the spin-0 unparticle case. For the value, $M_{D}=2 \mathrm{TeV}$, the cross section for LED graviton production is significantly higher in the tail than for the unparticle scenarios shown. The cross sections, however, scale with $M_{D}$ or $\Lambda_{\mathcal{U}}$ which allows the cross section from either of the two models to be larger or smaller than the other. Therefore, as pointed out in [16], it is important to also study the shape of the signal in order to understand which new physics model is more compatible with a possible mono-jet excess at the LHC.

\section{Virtual Exchange, Photon Pair Production}

The Pythin8 processes related to virtual $G$ exchange ffbar2gammagamma and gg2gammagamma are controlled by the program parameters LambdaT, CutOffmode, $t$, and for these processes CutOffmode $=1$ is not available. The corresponding unparticle processes have the same names, but reside in the different unparticle process category as listed in the appendix. They are controlled by the following parameters spinU, dU, LambdaU, lambda, with the two possible spin values $\operatorname{spinU}=0,2$. 


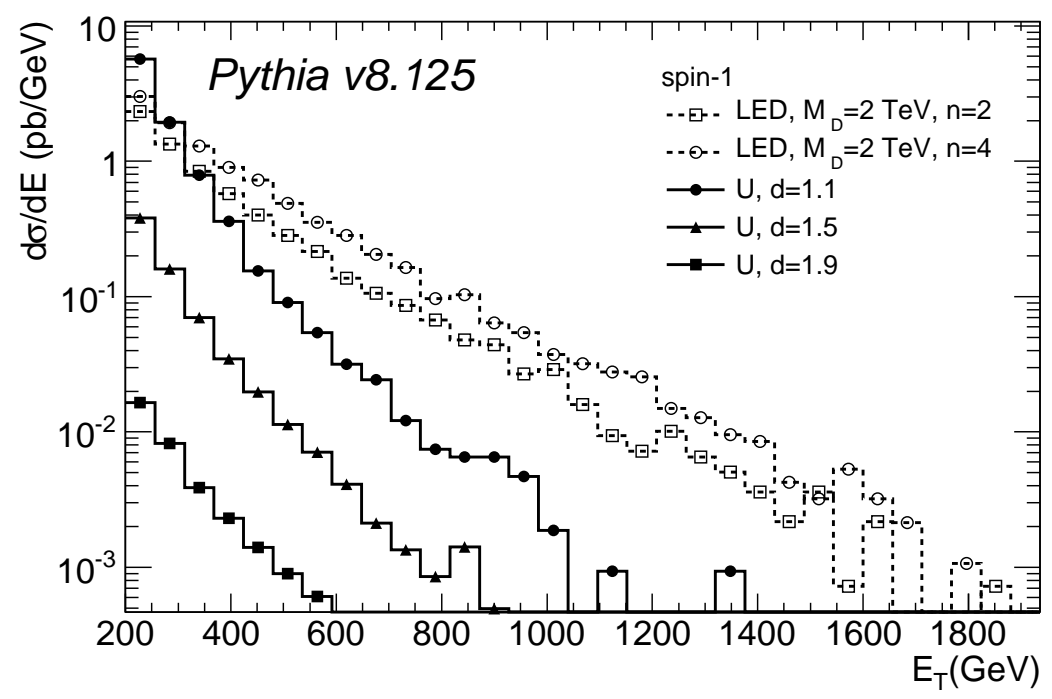

Figure 4: Differential cross sections as a function of the transverse jet energy for LED graviton emission and emission of spin-1 unparticles at the $\operatorname{LHC}(\sqrt{s}=14 \mathrm{TeV})$.

This section presents different characteristic results for di-photon production due to both virtual $\mathcal{U}$ and $G$ exchange. The validation of these processes is based on the results presented in $[6,7,9,11,13]$ and similar selection cuts as well as model parameter values have been chosen for easy comparison.

\subsection{Virtual Graviton Exchange}

Fig. 5 shows the invariant mass distribution of the two photons produced by LED graviton exchange, with and without using the form factor in Eq. (12). In accordance with [6] the mass scale value, $\Lambda_{H}=2.5 \mathrm{TeV}$, has been used ${ }^{8}$. The results using the form factor are shown for a number of different values of $n$. For virtual graviton exchange the renormalisation scale has been set to $\mu^{2}=\hat{s}$ (CutOffMode $=2$, renormScale2 $=4$ ). The characteristic effects from the form factor are visible, in particular the softening of the invariant mass distribution. As expected from Eq. (12), the distributions decrease faster at large $M_{\gamma \gamma}$ values for large $n$ and they intersect at $M_{\gamma \gamma}=\Lambda_{T}$, which for example in Fig. 5 corresponds to $2.8 \mathrm{TeV}$. The study also showed the expected behaviour that the graviton contribution becomes significant at larger $M_{\gamma \gamma}$ values with increased values of $\Lambda_{T}$.

\subsection{Virtual Unparticle Exchange}

The results related to $\gamma \gamma$ production at the LHC from virtual unparticle exchange have been compared with [9]. Cuts have been applied in all cases on the photon rapidity, $\left|y^{\gamma}\right|<2.5$, and transverse momentum $p_{T}^{\gamma}>40 \mathrm{GeV}$. For the invariant mass distributions the cut on the polar angle, $\left|\cos \theta_{\gamma}\right|<0.8$, has been used and for the angular and rapidity distributions only the invariant mass region, $600<M_{\gamma \gamma}<900 \mathrm{GeV}$, has been considered. Fig. 6 and 7 show

\footnotetext{
${ }^{8}$ The value using the $\Lambda_{H}$ convention was simply translated to, $\Lambda_{T}=2.8 \mathrm{TeV}$, as described in section 2.1 .
} 


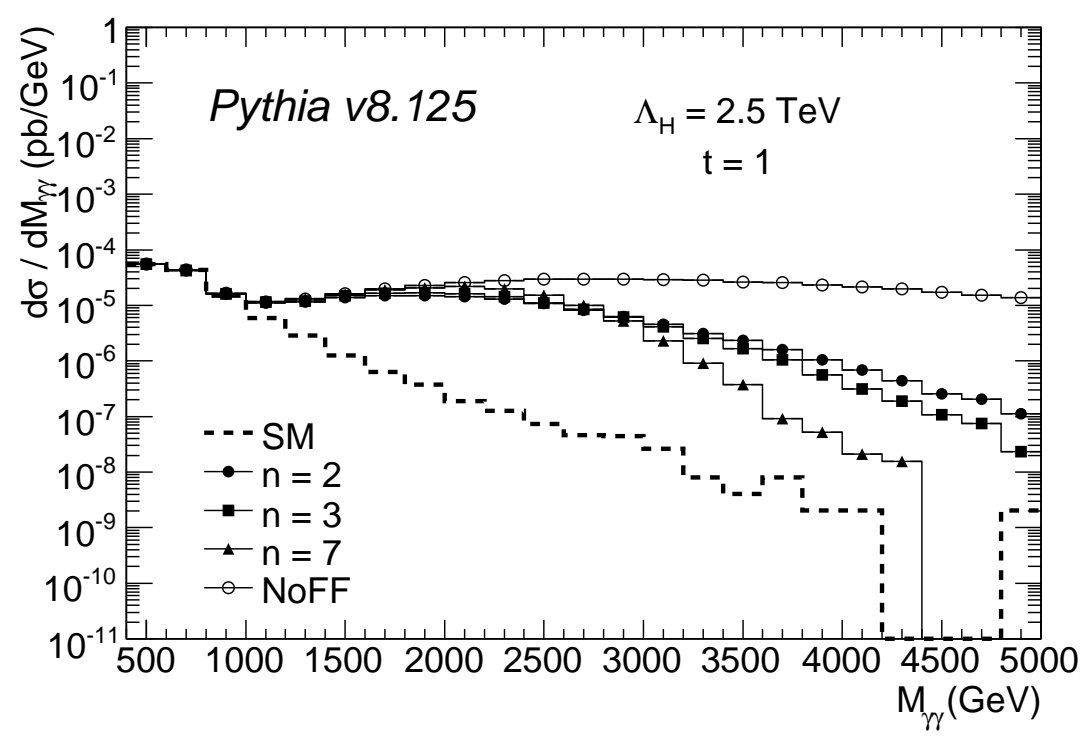

Figure 5: The di-photon invariant mass spectrum for LED graviton exchange at the LHC using $\Lambda_{H}=2.5$ $\mathrm{TeV}$. The results are produced with and without using the form factor, assuming various values of $n$.

the differential cross section as a function of $M_{\gamma \gamma}$ and $\cos \theta_{\gamma}^{*}$ respectively, for spin-0 unparticles, where $\theta_{\gamma}^{*}$ corresponds to the polar angle in the centre-of-mass frame of the photons. The invariant mass distribution is shown for three different $\lambda$ values and the signal decreases in the expected way with smaller coupling constants. The characteristic angular shape of the signal is visible and the results are found to be in good agreement with the literature listed above. In addition, the effects on various kinematic distributions from using different PDFs have been studied by using again the PDF sets MRST2001, CTEQ6L, GRV94L and the results showed only small differences.

\section{Virtual Exchange, Lepton Pair Production}

The available processes in PyTHIA8 with virtual graviton exchange leading to lepton pairs in the final state (Drell-Yan) correspond to $f f b a r 2 l l$ and gg2ll. The relevant program parameters are LambdaT, CutOffmode, $t$, where CutOffmode $=1$ is not available. Similar to the $\gamma \gamma$ case, the corresponding unparticle processes have the same names, but reside in the different unparticle process category as listed in the appendix. They are controlled by the parameters spinU, dU, LambdaU, lambda, gXX, gXY, with two unparticle spin values available spinU $=1,2$.

In this section we present a number of results obtained with PYTHIA8 used to validate the implementation of these processes as well as to quantify their characteristic behaviour. The processes are mainly validated against the results presented in $[6,7,11,13]$ and the choices of model parameter values and several selection criteria have also been based on these papers. We mainly focus the study on lepton pair production at the LHC. However, since most of the literature related to effects from chiral spin-1 unparticle couplings is available for $e^{+} e^{-}$-collisions we also present some validation results in that context. 


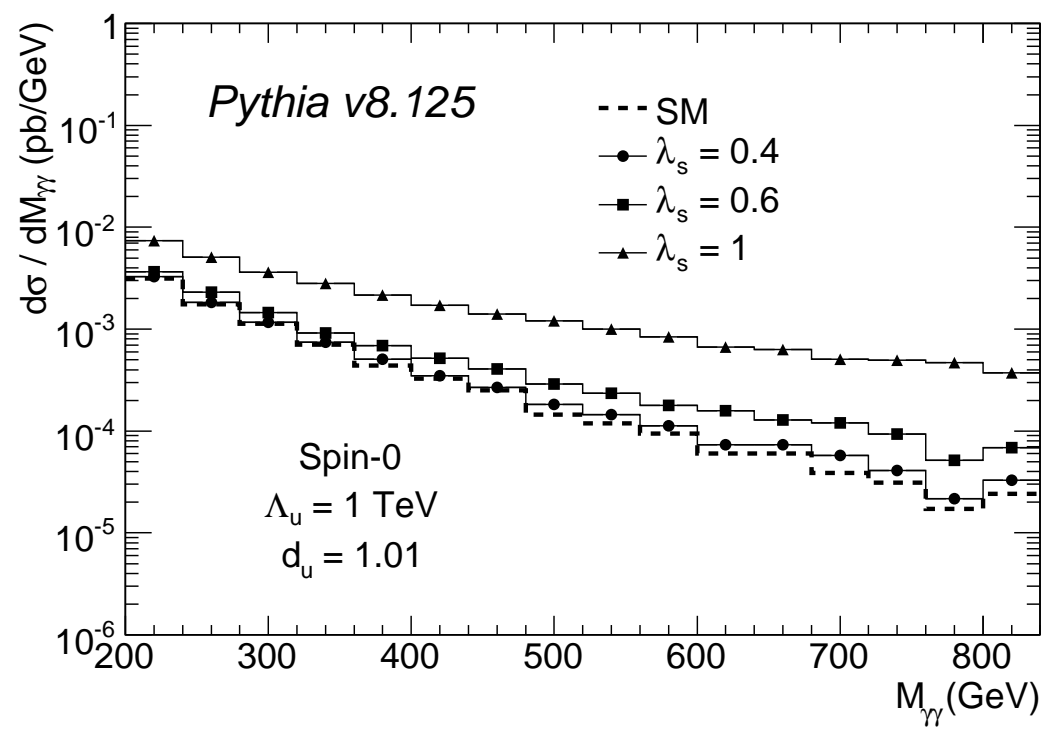

Figure 6: Invariant mass distribution of the di-photon system from spin-0 unparticle exchange at the LHC $(\sqrt{s}=14 \mathrm{TeV})$ for different values of the $\lambda_{s}$ and $\lambda_{t}$ couplings.

\subsection{Virtual Graviton Exchange}

In addition to the di-photon production at the LHC, discussed in Sec. 4, the production of two leptons can be influenced by the effects of virtual graviton exchange. For this reason, the invariant mass distribution of the two final-state leptons has been studied for a large number of different parameter settings of the LED model and agreement with the reference literature has been found. As an example, Fig. 8 shows the differential cross section as a function of the invariant mass of the lepton pair for the cut-off scale, $\Lambda_{H}=2.5 \mathrm{TeV}$, in scenarios with $n=3$. As for the $\gamma \gamma$ process the mass scale value in the common $\Lambda_{H}$ convention is used. In contrast to the $\gamma \gamma$ case the results are given for a number of different $t$ parameter values at a fixed $n$. The results show the strongly $t$-dependent increase of the cross section at high masses for the LED scenarios with respect to the SM. As expected from Eq. (12), the differential cross section converges towards the SM prediction for decreasing values of $t$, while for larger $t$ values, e.g. $t=2.0$, it approaches the LED scenarios where the form factor is not used (NoFF). In addition, the dependence of the cross section on the number of extra dimensions, $n$, has been studied and a similar behaviour to the di-photon production (c.f. Fig. 5) has been found as well as a good agreement with the results in the literature.

\subsection{Virtual Unparticle Exchange}

Similar to the virtual graviton exchange discussed above, the virtual exchange of unparticles can modify the invariant mass spectrum and the angular distribution of final-state lepton pairs at the LHC as well as in $e^{+} e^{-}$-collisions. The PYTHIA 8 results from various settings of the unparticle model parameters have been compared to the original literature and again good agreement has been found with the results for $p \bar{p}$ collisions at the Tevatron as presented in [7].

Fig. 9 shows the differential Drell-Yan cross section at the LHC as a function of the invariant 


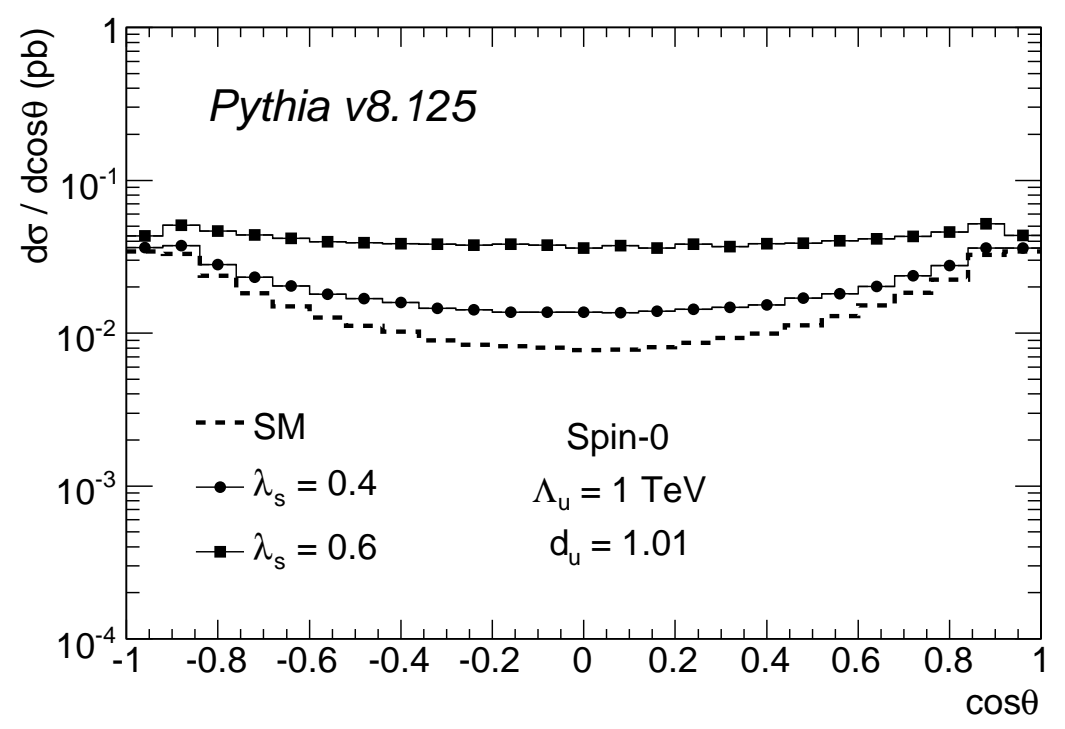

Figure 7: Angular distribution, $\cos \theta_{\gamma}^{*}$, of the photons from spin-0 unparticle exchange at the LHC $(\sqrt{s}=14 \mathrm{TeV})$.

mass of the lepton pair for various values of $d_{\mathcal{U}}$, assuming unparticles with spin-1 and vector-like 4 -fermion interactions. A cut on the lepton pair rapidity $(|y|<1)$ has been applied, which ensures that only central leptons are selected. As expected, the characteristic increase of the differential cross section due to the unparticle signal is more prominent for small values of $d_{\mathcal{U}}$, e.g. $d_{\mathcal{U}}=1.3$, both above and below the $Z$ pole.

The possibility of chiral spin-1 unparticle couplings could imply various effects, both affecting the invariant mass as well as the angular distribution of the final-state lepton pair. These effects have mainly been studied for $e^{+} e^{-}$-collisions in the literature and we therefore include the angular differential cross section obtained by PYTHIA8 for the SM and unparticle model, using various $d_{\mathcal{U}}$ values, in $e^{+} e^{-}$-collisions with $\sqrt{s}=200 \mathrm{GeV}$. Fig. 10 shows the angular distributions for $L L+R R$ (left) and $L R+R L$ (right) interactions, where the strong impact of a chiral coupling on the angular distribution is demonstrated. The parameter values $\Lambda_{\mathcal{U}}=1 \mathrm{TeV}$ and $\lambda=1$ have been used. The angle $\theta$ is defined as the emission angle of the $\mu^{-}$relative to the momentum vector of the incoming electron.

The angular dependence of the differential cross section can be investigated by studying the forward-backward asymmetry which is commonly defined as

$$
A_{F B}=\frac{N_{F}-N_{B}}{N_{F}+N_{B}}
$$

where $N_{F}$ and $N_{B}$ are the numbers of forward $(\cos \theta>0)$ and backward $(\cos \theta<0)$ produced events, respectively. At hadron colliders the determination of the angle $\theta$ is more complicated, since the direction of the incoming fermion is unknown. However, $A_{F B}$ is foreseen to be studied at the LHC [19] exploiting the fact that in annihilations of a valence quark and sea anti-quark the valence quark has, on average, a larger momentum than the sea quark. The resulting longitudinal momentum of the di-lepton system approximates the quark direction and, therefore, the angle 


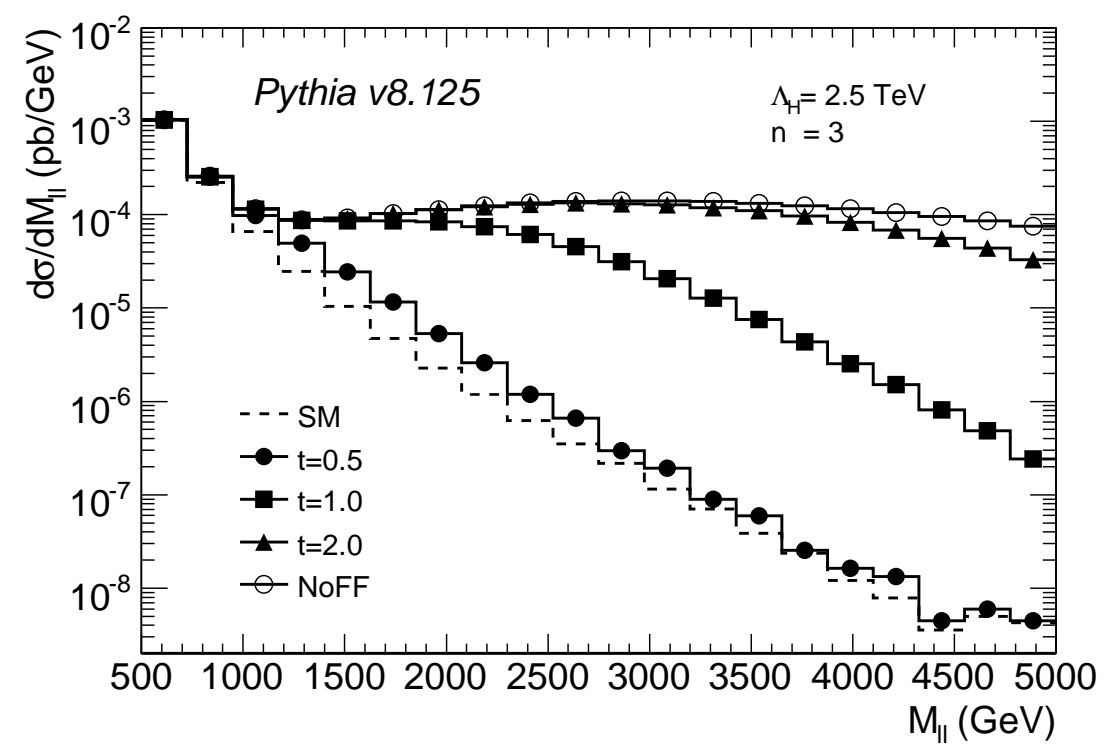

Figure 8: Differential cross section for the production of two charged leptons at the LHC as a function of their invariant mass. The results are shown for various LED scenarios with $n=3$ and $\Lambda_{H}=2.5 \mathrm{TeV}$.

$\theta$ between the lepton and quark in the di-lepton rest frame can be calculated. For the results presented here we use this method at generator level. In addition, we require, $|y|>1$, for the lepton rapidity and that at least one electron is in the central region, $|\eta|<2.5$.

In Fig. 11 the estimate of $A_{F B}$, using the method described above, is shown for the first time together with effects from unparticle exchange. The two plots show the invariant mass range 50 to $200 \mathrm{GeV}$ (left) and 200 to $1600 \mathrm{GeV}$ (right) respectively for various unparticle scenarios and the SM. It can been seen that certain unparticle scenarios $\left(e . g . \quad d_{\mathcal{U}}=1.3\right)$ lead to large deviation from the SM even at mass values below the $Z$ pole which are already investigated in detail by past experiments [20]. However, scenarios (e.g. $\left.d_{\mathcal{U}}=1.8\right)$ resulting in sizable effects only at larger mass values also exist, i.e. they are consistent with the SM in the already studied region at smaller masses.

Experimental effects, in particular statistics, will pose a significant challenge for the reconstruction of $A_{F B}$. A detailed experimental study is therefore needed to estimate the feasibility. However, in the case of an observation of a signal in the high mass tail (c.f. Fig. 8), a study of $A_{F B}$ as a function of the invariant mass could be of interest in order to confirm a non-SM content in the Drell-Yan spectrum.

\section{Full Event Simulation}

In order to allow for easy comparison with results in the literature as well as to clearly illustrate the effects related to the model parameters, the results from the various processes presented in this paper have been considered at the level of the hard process.

The processes are, however, integrated within the PYTHIA8 framework of parton showers, underlying event activity, hadronisation and unstable particle decays. These steps both increase the complexity of the events as well as affect their kinematic properties and are normally required 


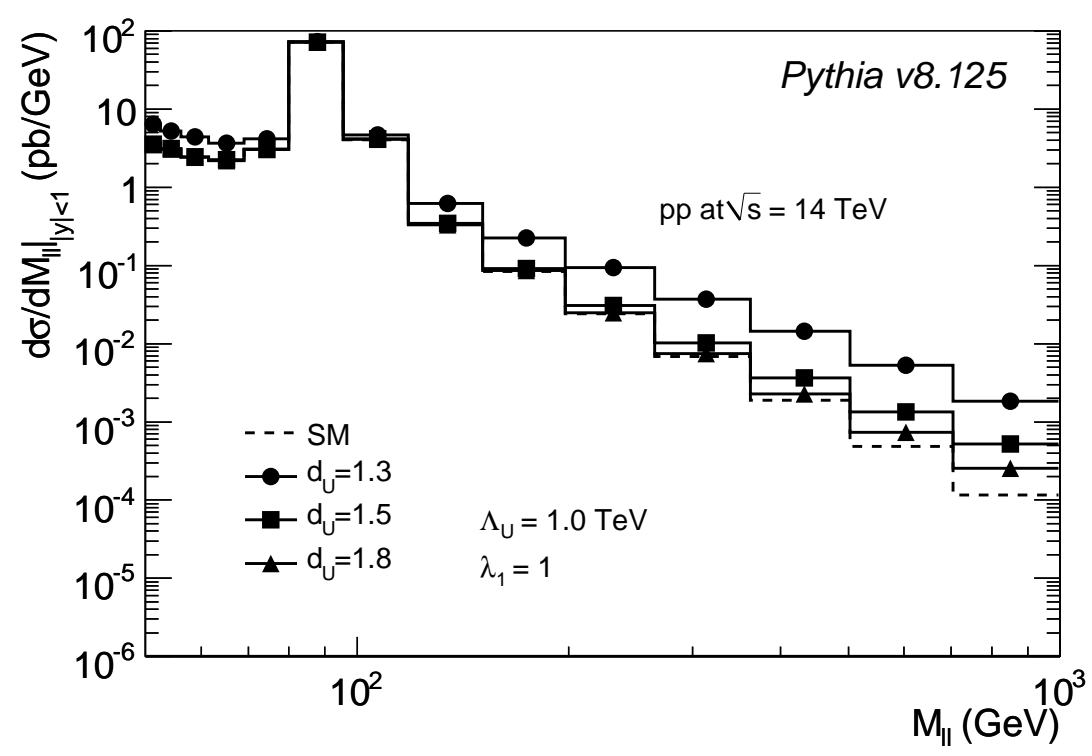

Figure 9: Differential cross section for the production of two charged leptons as a function of their invariant mass. The results are shown for scenarios with unparticles using different values of $d_{\mathcal{U}}$ at the LHC.

for proper comparisons between simulated phenomena and experimental data.

As an example, Fig 12 shows how initial state radiation (ISR) affects the $p_{T}$ in $G+j e t$ production. At leading order the $G$ and $j e t$ have exactly balanced $p_{T}$, but additional radiation from ISR, or other higher order effects, breaks this balance. Fig 12 shows the $p_{T}$ difference between the $G$ and the $j e t$ after ISR, relative to the original jet $p_{T}$ in the hard process. This is shown as a function of the jet $p_{T}$ at the hard process level and for the different $G+j e t$ sub-processes separately. As seen in the plot, ISR affects the sub-processes noticeably, however, to different extent and the imbalance decrease for higher $p_{T}$ jets. This clearly illustrates the importance of building the processes into the simulation of the full event structure in Pythia in order to model such effects.

\section{Conclusions}

Both large extra dimensions as well as unparticle models have become very popular among possible extensions of the SM. Many of the main processes related to LED searches have analogous processes in the unparticle scenario, which are often more general in terms of model parameters. A common way to implement the similar processes has been found within the Monte Carlo generator PYTHIA8, where only a few translations of model dependent constants are necessary. In addition to technical benefits, the common implementation makes comparisons between the processes from the two models consistent and simplifies studies of how differences at the process level for a certain final state can affect the measured observables. The processes can be used together with several options for the treatment of the UV region of the effective theory. In this paper, the options related to the individual processes have been discussed together with results obtained by PYTHIA8. In addition to providing references for the PYTHIA8 program, the results 

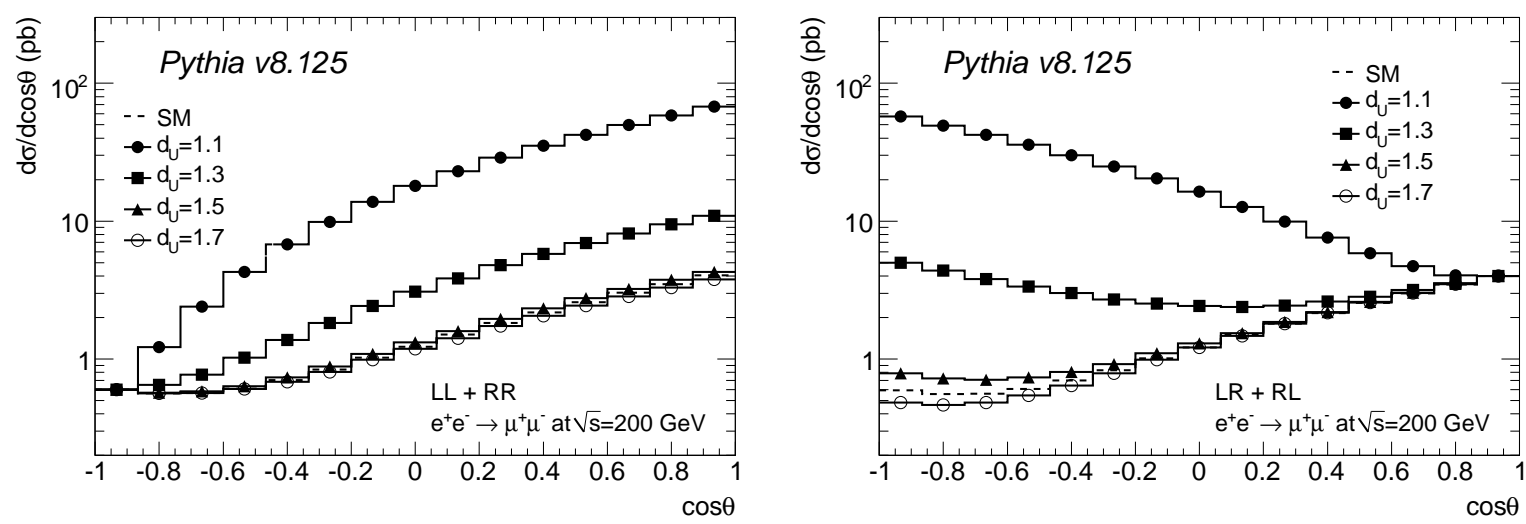

Figure 10: Angular distribution for $e^{+} e^{-} \rightarrow \mu^{+} \mu^{-}$including unparticle exchange, for various values of $d_{\mathcal{U}}$ at $\sqrt{s}=200 \mathrm{GeV}$, assuming $L L+R R$ (left) and $L R+R L$ (right) 4-fermion interactions.
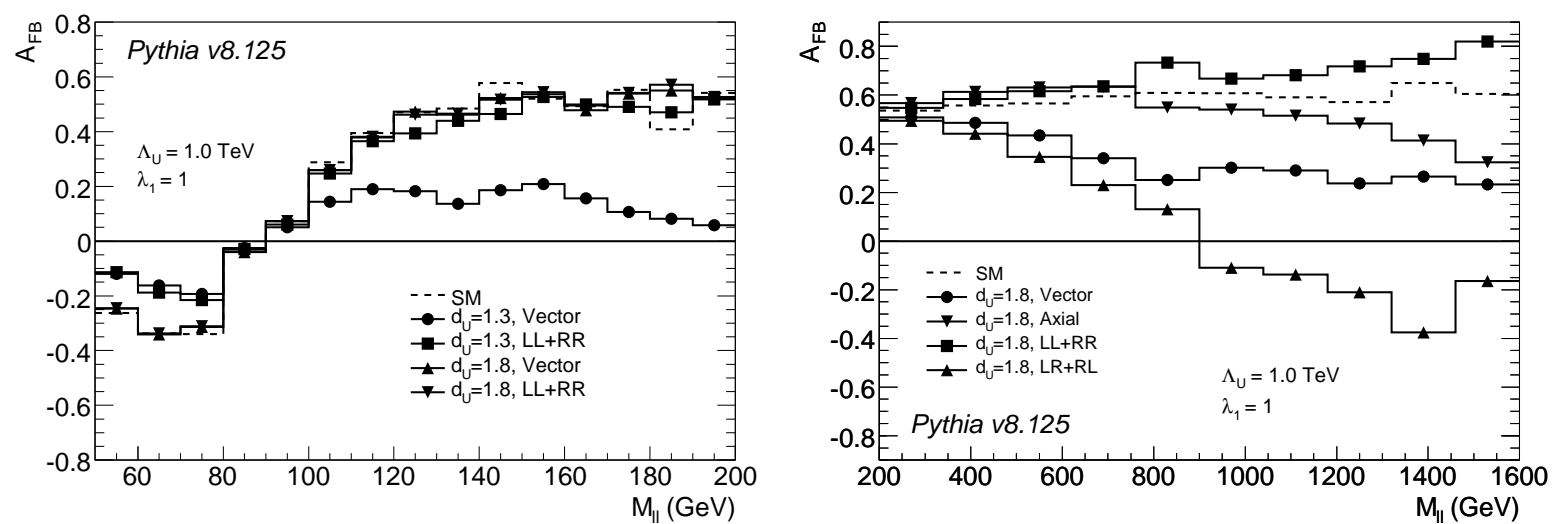

Figure 11: $A_{F B}$ as a function of the invariant mass of the lepton pair at the LHC. The results are shown for $d_{\mathcal{U}}=1.3,1.8$ with vector-like and $L L+R R$ unparticle interactions (left) as well as for $d_{\mathcal{U}}=1.8$ with various chiral interactions (right). In both figures spin-1 unparticle exchange is assumed.

illustrate characteristic effects of the model parameters and various key features of the different kinematic distributions. The results presented in this paper have been focused on mono-jet, di-photon and di-lepton final states at the LHC.

\section{Acknowledgment}

SA would like to express special thanks to Wei-Yee Keung (UIC) for providing the formulae related to the scalar $Z \mathcal{U}$ process and to Henrik Jansen (RWTH) for related helpful feedback. This work was funded in the UK by STFC and by the German Research Foundation (DFG) in the Collaborative Research Centre (SFB) 676 "Particle, Strings and the Early Universe" located in Hamburg. This work was supported in part by the EU Marie Curie Research Training Network "MCnet", under contract number MRTN-CT-2006-035606. 


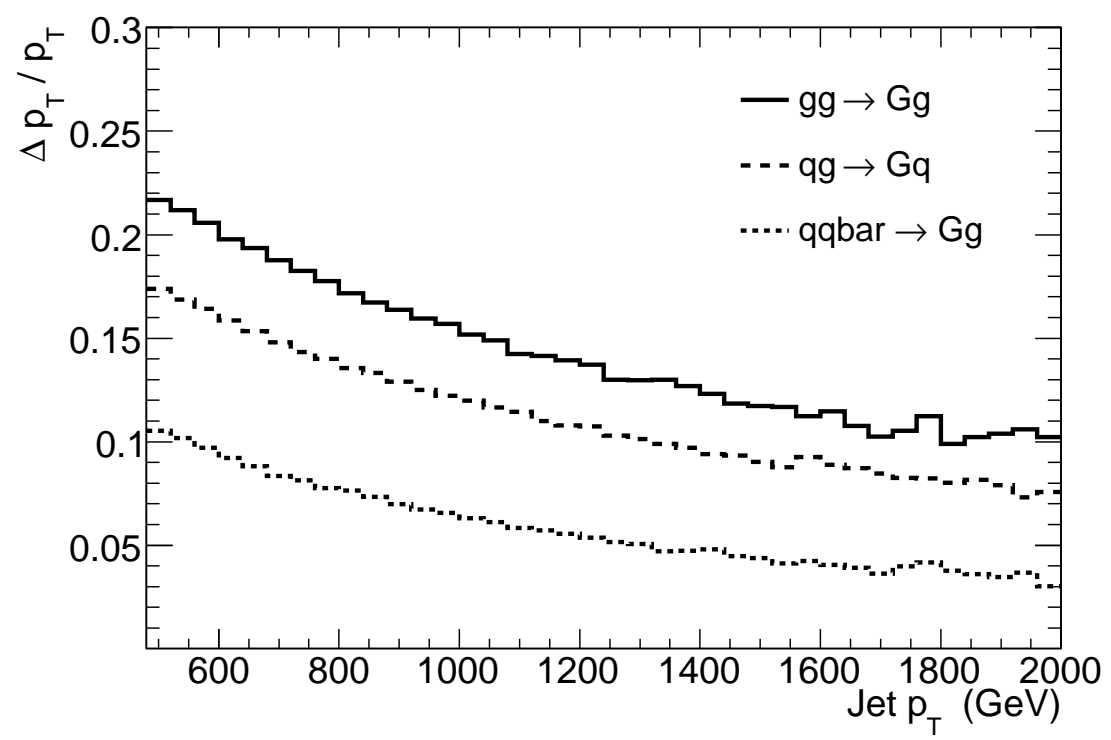

Figure 12: Relative $p_{T}$ difference between the graviton and jet due to initial state radiation. The results are shown for $G+$ jet production with $n=6$ and $M_{D}=4 \mathrm{TeV}$.

\section{Appendix - $\mathcal{U} / G$ Specific Parameters in Pythia8}

In the PYTHIA8 program the $\mathcal{U} / G$ processes are turned on/off by the following parameters,

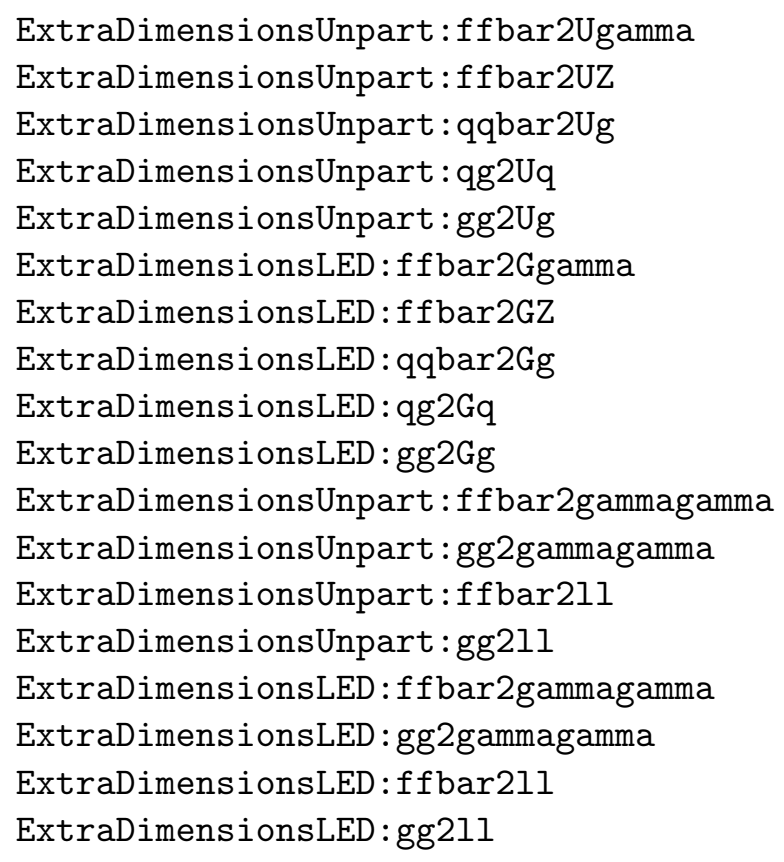

- Real $\mathcal{U}$ emission, $f \bar{f} \rightarrow \mathcal{U}+\gamma$.

- Real $\mathcal{U}$ emission, $f \bar{f} \rightarrow \mathcal{U}+Z$.

- Real $\mathcal{U}$ emission, $q \bar{q} \rightarrow \mathcal{U}+g$.

- Real $\mathcal{U}$ emission, $q g \rightarrow \mathcal{U}+q$.

- Real $\mathcal{U}$ emission, $g g \rightarrow \mathcal{U}+g$.

- Real $G$ emission, $f \bar{f} \rightarrow G+\gamma$.

- Real $G$ emission, $f \bar{f} \rightarrow G+Z$.

- Real $G$ emission, $q \bar{q} \rightarrow G+g$.

- Real $G$ emission, $q g \rightarrow G+q$.

- Real $G$ emission, $g g \rightarrow G+g$.

- Virtual $\mathcal{U}$ exchange, $f \bar{f} \rightarrow\left(\mathcal{U}^{*}\right) \rightarrow \gamma \gamma$.

- Virtual $\mathcal{U}$ exchange, $g g \rightarrow\left(\mathcal{U}^{*}\right) \rightarrow \gamma \gamma$.

- Virtual $\mathcal{U}$ exchange, $f \bar{f} \rightarrow\left(\mathcal{U}^{*}\right) \rightarrow \ell \bar{\ell}$.

- Virtual $\mathcal{U}$ exchange, $g g \rightarrow\left(\mathcal{U}^{*}\right) \rightarrow \ell \bar{\ell}$.

- Virtual $G$ exchange, $f \bar{f} \rightarrow\left(G^{*}\right) \rightarrow \gamma \gamma$.

- Virtual $G$ exchange, $g g \rightarrow\left(G^{*}\right) \rightarrow \gamma \gamma$.

- Virtual $G$ exchange, $f \bar{f} \rightarrow\left(G^{*}\right) \rightarrow \ell \bar{\ell}$.

- Virtual $G$ exchange, $g g \rightarrow\left(G^{*}\right) \rightarrow \ell \bar{\ell}$.

The model parameters related to the LED graviton processes are specified by, 
ExtraDimensionsLED: $\mathrm{n}$

ExtraDimensionsLED:MD

ExtraDimensionsLED: LambdaT

ExtraDimensionsLED : CutOffmode

ExtraDimensionsLED: t
- Number of large extra dimensions, $n$.

- Fundamental scale of $D$ dimensional gravity, $M_{D}$.

- Cut-off scale for virtual $G$ exchange, $\Lambda_{T}$.

- This parameter specifies the treatment of the high energy contributions. Possible values are 0 to 3 .

- Form factor parameter, $t$.

The model parameters related to the unparticle processes are specified by,

ExtraDimensionsUnpart: spinU

ExtraDimensionsUnpart: dU

ExtraDimensionsUnpart: LambdaU

ExtraDimensionsUnpart: lambda

ExtraDimensionsUnpart:gXX

ExtraDimensionsUnpart:gXY
- The unparticle spin. Possible values are, 0, 1 or 2.

- Scale dimension parameter, $d_{\mathcal{U}}$.

- Renormalization scale, $\Lambda_{\mathcal{U}}$.

- Coupling to the SM fields, $\lambda$.

- Options for the LL/RR helicity contributions, $g_{X X}$. This parameter is only relevant for the spin-1 $\ell \bar{\ell}$ process, where the available options 0,1 and 2 correspond to the helicity amplitudes being multiplied by a factor 1,0 or -1 .

- Options for the LR/RL helicity contributions, $g_{X Y}$. This parameter is only relevant for the spin- $1 \ell \bar{\ell}$ process, where the available options 0,1 and 2 correspond to the helicity amplitudes being multiplied by a factor 1,0 or -1 .

- This parameter specifies the treatment of the high energy contributions. Possible values are 0 or 1 .

More details on the meaning of the model parameters are given in the text.

\section{Appendix - Scalar Unparticle Production, $f \bar{f} \rightarrow Z \mathcal{U}$.}

The relevant scalar unparticle interaction terms for this process are given by,

$$
\mathcal{L} \supset \frac{O_{\mathcal{U}}}{\Lambda_{\mathcal{U}}^{d_{\mathcal{U}}-1}} \bar{f}\left(\lambda_{0} 1+\lambda_{0}^{\prime} i \gamma_{5}\right) f
$$

Due to $\mathrm{CP}$ conservation, the two couplings $\lambda_{0}$ or $\lambda_{0}^{\prime}$ are only considered separately. Since the matrix element expression of the two cases becomes identical in the absence of any interference with the SM, only one common $\lambda$ value is used for the implementation in accordance with Eq. (3). These couplings are usually suppressed by a quark mass factor, because of the chirality flipping, and are therefore expected to be small in the scalar unparticle case. However, in the phenomenological spirit of this paper the choice of restricting model parameter values is left to the user.

As described in [5], the cross section for the, $f \bar{f} \rightarrow Z \mathcal{U}$, process is given by,

$$
\frac{d^{2} \sigma}{d P_{\mathcal{U}}^{2} d t}=\frac{|\bar{M}|^{2}}{16 \pi \hat{s}^{2}} \frac{A_{d_{\mathcal{U}}}}{2 \pi \Lambda_{\mathcal{U}}^{2}}\left(\frac{d P_{\mathcal{U}}^{2}}{\Lambda_{\mathcal{U}}^{2}}\right)^{d_{\mathcal{U}}-2}
$$


where $|\bar{M}|$ is the colour and spin averaged matrix element and $P_{\mathcal{U}}^{2}$ is the invariant mass of the unparticle, $0 \leq P_{\mathcal{U}}^{2} \leq\left(\sqrt{s}-m_{Z}\right)^{2}$. The matrix element is given by [21],

$$
\begin{array}{r}
|\bar{M}|^{2}=\frac{1}{4 N_{c}}\left(\frac{e^{2}}{\sin ^{2} \theta_{W} \cos ^{2} \theta_{W}}\right)\left(g_{L, q}^{2}+g_{R, q}^{2}\right) \lambda^{2}|A|^{2} \\
|A|^{2}=4\left[-\frac{s}{t}-\left(1-\frac{m_{Z}^{2}}{t}\right)\left(1-\frac{P_{\mathcal{U}}^{2}}{t}\right)-\frac{s}{u}\right. \\
\left.-\left(1-\frac{m_{Z}^{2}}{u}\right)\left(1-\frac{P_{\mathcal{U}}^{2}}{u}\right)+2\left(1-\frac{P_{\mathcal{U}}^{2}}{t}\right)\left(1-\frac{P_{\mathcal{U}}^{2}}{u}\right)\right]
\end{array}
$$

with $g_{L, d}=-\frac{1}{2}+\frac{1}{3} \sin ^{2} \theta_{W}, g_{R, d}=+\frac{1}{3} \sin ^{2} \theta_{W}, g_{L, u}=\frac{1}{2}-\frac{2}{3} \sin ^{2} \theta_{W}$ and $g_{R, u}=-\frac{2}{3} \sin ^{2} \theta_{W}$. Regarding the amplitude formula, $|A|^{2}$, the first two terms inside the square brackets result from the $t$-channel, the following two terms from the $u$-channel and the last term from the interference.

In the same way as for the other spin versions of this process, the process where a photon is emitted together with an unparticle has been obtained by the following photon limit of the $Z$ matrix element,

$$
\begin{array}{r}
m_{Z} \rightarrow 0 \\
\frac{g_{L}^{2}+g_{R}^{2}}{2} \rightarrow Q^{2} \\
\frac{1}{\sin \theta_{W} \cos \theta_{W}} \rightarrow 1
\end{array}
$$

where $Q$ is the electric charge of the incoming fermions. 


\section{References}

[1] N. Arkani-Hamed, S. Dimopoulos and G. Dvali, Phys. Lett. B429 (1998) 263;

I. Antoniadis, N. Arkani-Hamed, S. Dimopoulos and G. Dvali, Phys. Lett. B436 (1998) 257.

[2] C. Amsler et al. (Particle Data Group), Phys. Lett. B667 (2008) 1 (URL: http://pdg.lbl.gov). Review of Extra dimensions, by G.F. Giudice and J.D. Wells, together with references therein.

[3] H. Georgi, Phys. Rev. Lett. 98 (2007) 221601.

[4] T. Sjöstrand, S. Mrenna and P. Skands, JHEP 05 (2006) 026;

T. Sjöstrand, S. Mrenna and P. Skands, Comp. Phys. Comm. 178 (2008) 852.

[5] S. Ask, Eur. Phys. J. C60 (2009) 509.

[6] J. Hewett, T. Rizzo, JHEP 0712 (009) 2007.

[7] K. Cheung, W.-Y. Keung and T.-C. Yuan, Phys. Rev. D76 (2007) 055003.

[8] H. Georgi and Y. Kats, arXiv:0904.1962 (2009).

[9] M.C. Kumar, P. Mathews, V. Ravindran, and A. Tripathi, Phys. Rev. D77 (2008) 055013.

[10] G. Cacciapaglia, G. Marandella and J. Terning, JHEP 0902 (2009) 049.

[11] G.F. Giudice, R. Rattazzi and J.D. Wells, Nucl. Phys. B544 (1999) 3.

[12] L. Randall and R. Sundrum, Phys. Rev. Lett. 83 (1999) 3370.

[13] G.F. Giudice, T. Plehn and A. Strumia, Nucl. Phys. B706 (2005) 455.

[14] E.A. Mirabelli, M. Perelstein and M.E. Peskin, Phys. Rev. Lett. 82 (1999) 2236.

[15] T. Han, J.D. Lykken, R.-J. Zhang, Phys. Rev. D59 (1999) 105006.

[16] T. G. Rizzo, Phys. Lett. B665 (2008) 361.

[17] H. Georgi, Phys. Lett. B650 (2007) 275.

[18] L. Vacavant and I. Hinchliffe, J. Phys. G: Nucl. Part. Phys. 27 (2001) 1839.

[19] ATLAS Collaboration, G. Aad et al., CERN-OPEN-2008-020, arXiv:0901.0512v1 [hep-ex].

[20] C. Amsler et al. (Particle Data Group), Phys. Lett. B667 (2008) 1 (URL: http://pdg.lbl.gov). Review of Electroweak model and constraints on new physics, by J. Erler and P. Langacker, together with references therein.

[21] W.-Y. Keung, private communications. 